\author{
Chrischta Ganz
}

Bei den Schweizer Naturheilheilpraktikern und Naturheilpraktikerinnen ereignet sich aufgrund der Definition des Berufs «eidgenössisch diplomierte(r) Naturheilpraktiker/in» (in den Fachrichtungen Traditionelle Chinesische Medizin (TCM), Traditionelle Europäische Naturheilkunde (TEN), Homöopathie oder Ayurvedamedizin) berufspolitisch momentan sehr viel. Aus diesem Grund werden in drei Ausgaben der SchweizeriSCHEN ZEITSCHRIFT FÜR GANZHEITSMEDIZIN einige Schweizer Ausbildungsorte für Naturheilpraktiker/innen vorgestellt. Es sind bekannte und etablierte Schweizer Schulen, die in der Schweizer Bildungslandschaft von vielen weiteren, teils kleineren Heilpraktikerschulen sowie diversen Ausbildungsstätten für Komplementärtherapien (KT) ergänzt werden.

Im ersten Teil wird die Akademie für ganzheitliche Medizin, Naturheilkunde TEN und Psychosomatik QuintaMed (Winterthur) (www.quintamed.ch) vorgestellt. Im zweiten Teil folgen die Heilpraktikerschule (HPS) (Luzern) und die Akademie für $\mathrm{Na}$ turheilkunde (ANHK) (Basel)/Sake (Bern), und im dritten Teil werden die Schule für angewandte Naturheilkunde (Zürich), die Paramed (Zug) und die Bodyfeet-Schule (Thun, Rapperswil) vorgestellt.

Generell dauern die Ausbildungen an den meisten Schulen einige Jahre, können in Modulen berufsbegleitend absolviert werden und beinhalten neben schulmedizinischen Grundlagen das spezifische naturheilkundliche Wissen sowie manuelle und energetische Methoden.

\title{
Naturheilpraktikerschulen der Schweiz - Teil 1
}

\section{Begriff «Naturheilkunde»}

Um Missverständnissen vorzubeugen, verwendet die Autorin den Begriff «Naturheilkunde/Naturheilpraktiker/in» für die Alternativmedizin (AM), wie sie heute in der Schweizerischen Berufspolitik verwendet wird [1]. Da der Begriff «Naturheilkunde» in der Berufsbezeichnung der «Naturheilpraktiker/ innen» verwendet wird, wird dieser hier noch vertieft:

In der Schweiz hat der Begriff der Naturheilkunde eine lange Tradition. Mit Naturheilkunde bezeichnete man in Europa die klassische Naturheilkunde, die sich im 20. Jahrhundert als Reaktion bzw. Alternative zur nur noch wissenschaftlich orientierten und abstrakten Schulmedizin entwickelte. Das Wissen der Naturheilkunde ist jedoch schon viel älter, geht bis zu den Anfängen der Menschheit zurück und hat je nach Fachrichtung geografisch andere Ursprünge [2]. Unabhängig von der Fachrichtung beinhaltet Naturheilkunde das systemische Denken, die traditionelle Heilkunst aus der jeweiligen Region, eine kulturell gewachsene Erfahrungsmedizin sowie eine ganzheitliche und individuelle Sicht auf Mensch und Natur (Naturphilosophie).

Einfach formuliert basiert Naturheilkunde auf den seit jeher wirkenden Lebensgesetzen und Naturkräften, die modifiziert für den Heilungsprozess eingesetzt werden, um die Selbstheilungskräfte zu aktivieren.

\section{Persönlichkeitsentwicklung}

Auch der Frage der Persönlichkeitsentwicklung während der Ausbildung wird im Rahmen dieser Schul- portraits nachgegangen. Die Naturheilpraktiker/innen haben in ihrem Praxisalltag das Glück, «viel» Zeit zur Verfügung zu haben, um auf den Patienten einzugehen - insbesondere im Vergleich mit schulmedizinischen Ärztinnen und Ärzten. Dies ist ein Segen (dass sich Patienten dafür bedanken, dass man ihnen zuhört, ist in der naturheilkundlichen Praxis an der Tagesordnung), aber auch eine grosse Aufgabe und Herausforderung, die Empathie, eigene Persönlichkeitsentwicklung sowie eine gute psychologische Ausbildung voraussetzen.

\section{Akademie für ganzheitliche Medizin, Naturheilkunde TEN und Psychosomatik QuintaMed, Winterthur}

Die QuintaMed befindet sich seit einem halben Jahr in der Stadt Winterthur. Durch den Anspruch an Räumlichkeiten angepasster Grösse und optimaler technischer Einrichtung für den Unterricht ist die Schule nach 12 Jahren von Hettlingen $\mathrm{ZH}$ nach Winterthur umgezogen (Abb. 1). Auch die Eröffnung eines

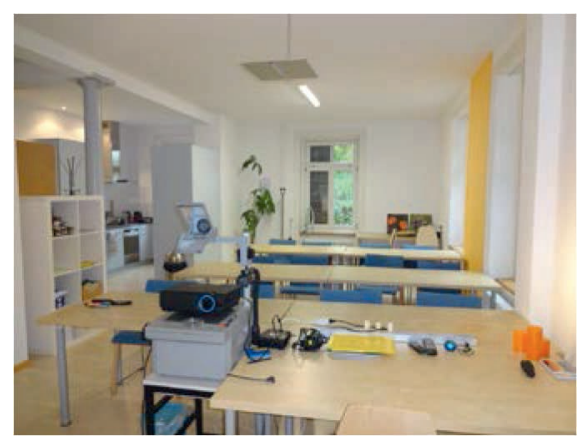

Abb. 1. Schulräumlichkeiten der QuintaMed.

\section{KARGER}

Fax +4976145207 14 Information@Karger.com www.karger.com (c) 2014 S. Karger GmbH, Freiburg
Chrischta Ganz

Praxis für Naturheilkunde

Friedhofstrasse 15, 8636 Wald ZH, Schweiz

naturheilpraxis@chrischtaganz.ch www.chrischtaganz.ch 
naturheilkundlichen Kompetenzzentrums (unter anderem für die Praktika der Studierenden) konnte mit dem Umzug umgesetzt werden. Einzelne Kursmodule werden nach wie vor in Hettlingen $\mathrm{ZH}$ oder auch in Teufen AR unterrichtet.

Die Schule wird von Heide-Dore Bertschi-Stahl als Schulleiterin (Abb. 2) und Thomas Bertschi in beratender Funktion geleitet. HeideDore Bertschi-Stahl zur Schule:

«Vor gut 12 Jahren gab es nur wenige naturheilkundliche Ausbildungsorte. Damals war die Ausbildung stark von den unterrichtenden Persönlichkeiten geprägt, und entsprechend gab es ein wildes 〈Durcheinander», was zu einer naturheilkundlichen Ausbildung gehört. Aus diesen Erfahrungen, die ich an verschiedenen Fachschulen im Gesundheitsbereich (als Dozentin, Prüfungsexpertin und Schulleiterin) machen konnte, war die Entwicklung der letzten 20 Jahre in der Ausbildungslandschaft gut mitzuverfolgen. Das hat mich bewogen, 2002 die Akademie QuintaMed für ganzheitliche Medizin, Naturheilkunde TEN und Psychosomatik zu gründen. Im gleichen Atemzug wurde die Ausbildung zum/r Naturheilpraktiker/in für Traditionelle Europäische Naturheilkunde (TEN) in Bausteine und Module aufgegleist. Diese Ausbildung hat sich in dieser Zeit mit kompetenten Dozenten für das neue Berufsbild entwickelt. Das kommt uns heute zugute.»

Die QuintaMed ist EduQua-zertifiziert und Mitglied im Verband Schweizerischer Privatschulen (VSP) sowie der Konferenz der Höheren Fachschulen Alternativmedizin (hfam).

Als kleinere Schule bringt sie den Vorteil der schlanken, flexiblen Struktur sowie einen vertrauten Umgang von Studierenden, Schulleitung, Dozenten und Sekretariat mit sich. Leitmotiv der QuintaMed sind die drei Ps «persönlich, professionell, praxisnah», nach denen sich der Unterricht und die tägliche Arbeit

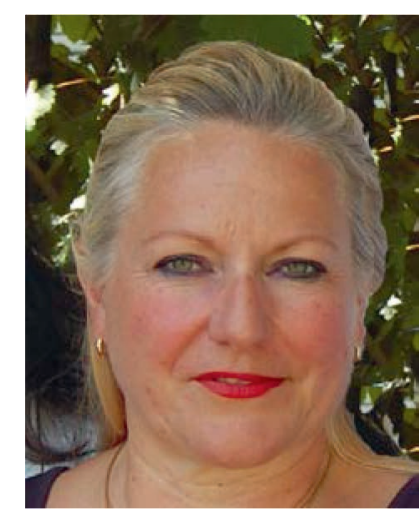

Abb. 2. Heide-Dore Bertschi Stahl, Schulleiterin der QuintaMed.

richten. Die QuintaMed hat sich mit ihrem Schwerpunkt Psychosomatik sowie im letzten Herbst mit dem ersten Kongress für Naturheilkunde einen guten Ruf geschaffen [3]. HeideDore Bertschi-Stahl ist ausserdem Mitautorin des Buchs «Grundlagen der Traditionellen Europäischen Naturheilkunde» [2].

Der Schwerpunkt der Akademie liegt klar bei der Fachrichtung TEN. Die Gesamtausbildung «Naturheilpraktiker/in TEN» wird in sieben Modulbausteine gegliedert. Die Erfahrungen und das Wissen der TEN werden mit den Erkenntnissen der modernen Medizin (teilweise auch mit den traditionellen Medizinalsystemen aus dem asiatischen Raum) zusammengeführt.

An der QuintaMed arbeiten schulmedizinisch ausgebildete Fachkräfte wie auch Naturheilpraktiker und Therapeuten aus der Praxis. Berührungsängste gibt es da keine - im Gegenteil, zum Wohle der Patienten wird viel Wert auf ein konstruktives Zusammenarbeiten von Schul- und Alternativmedizin gelegt.

Weitere Ausbildungen in ganzheitlicher Atem- und Körpertherapie sowie Manueller Lymphdrainage (MLD), Fussreflexzonentherapie, Ganzkörpermassage, Aromatherapie, Colon-Hydro-Therapie usw. ergänzen das Ausbildungsprogramm. Neben der Weiterbildung für erfahrene Therapeuten, Apotheker und
Drogisten werden auch Kurse für Laien angeboten.

Im Ambulatorium (Kompetenzzentrum QuintaSana in Winterthur und Praxis in Teufen AR) können die Studierenden gegen Ende ihrer Ausbildung ein professionell begleitetes Praktikum absolvieren. Die Praktikanten durchlaufen damit alle Stationen, die ein/e Naturheilpraktiker/in gesehen und praktisch erfahren haben sollte. So sind die Studierenden am Ende der Berufsausbildung gut gerüstet für eine eigene Praxistätigkeit. Die Möglichkeit eines Praktikums steht auch Studierenden anderer Ausbildungsorte offen.

\section{Naturheilkunde/ \\ Naturheilpraktiker/innen}

«Natura sanat» - Die Natur heilt ist das Motto der Schule. Freude an naturheilkundlichem Wissen und an der ganzheitlichen kompetenten Behandlung von Patienten, Sensibilität und Intuition sind Voraussetzungen für den Beruf, auf die viel Wert gelegt werden. Auffallend ist (generell an den meisten Schulen für AM und KT) der hohe Frauenanteil. Dazu HeideDore Bertschi-Stahls Gedanken: «Oft haben die Frauen das Wissen aus der Erfahrungs- und Volksmedizin weitergetragen. Durch Mutterschaft werden die Frauen eher für gesundheitliche Themen sensibilisiert.»

\section{Menschen- und}

\section{Persönlichkeitsentwicklung}

Die QuintaMed versteht sich nicht nur als reine Fachausbildung, sondern definiert die Menschen- und Persönlichkeitsbildung als Teil der Ausbildung. Eigene Prozesse und Selbsterfahrung während des Studiums werden gefördert, speziell in der «angewandten Psychologie», die ein wichtiger Teil der Ausbildung und Berufspraxis ist. Neben der Vermittlung des Fachwissens wird auch die Sensitivität für sich und andere geschult und gefördert - einerseits durch die Synergie von theoretischem und praktischem 
Erarbeiten eines Fachgebiets, andererseits durch bewusstes Schulen von Beobachtungsgabe, Sinneswahrnehmung, Mitgefühl sowie vernetztem Denken und Verantwortungsbe- wusstsein. So erfahren die Studierenden, dass die akuten und die chronischen Leiden den Menschen in seiner Ganzheit betreffen, und lernen, die Patienten empathisch zu begleiten.

\section{Literatur}

1 www.oda-am.ch.

2 Raimann C, Ganz C, Garvelmann F, Bertschi-Stahl H-D, Fehr-Streule R: Grundlagen der Traditionellen Europäischen Naturheilkunde. Bacopa, Schiedlberg, 2012.

3 www.naturheilkundekongress.ch. 